\title{
Urinary Bladder Nephrogenic Adenoma
}

National Cancer Institute

\section{Source}

National Cancer Institute. Urinary Bladder Nephrogenic Adenoma. NCI Thesaurus. Code C7415.

A metaplastic lesion of the urothelium found in the urinary bladder. It is characterized by the presence of aggregates of cuboidal or hobnail cells and represents a reaction of the urothelium to injury caused by instrumentation, surgery or calculi. 